\title{
Experimental and numerical investigation on cloud cavitating flow around an axisymmetric projectile near the wall with emphasis on the analysis of local cavity shedding
}

\author{
Chao Yu ${ }^{\mathrm{a}, \mathrm{b}}$, Yiwei Wang ${ }^{\mathrm{a}, \mathrm{b}, *}$, Chenguang Huang ${ }^{\mathrm{a}, \mathrm{b}}$, Tezhuan $\mathrm{Du}^{\mathrm{a}}$, Chang $\mathrm{Xu}^{\mathrm{a}}$, Jian Huang ${ }^{\mathrm{a}}$ \\ a Key Laboratory for Mechanics in Fluid Solid Coupling Systems, Institute of Mechanics, Chinese Academy of Sciences, No.15 Beisihuanxi Road, Beijing \\ 100190, China \\ b School of Engineering Science, University of Chinese Academy of Sciences, China
}

\section{A R T I C L E I N F O}

\section{Keywords:}

Unsteady cloud cavitation

Effect of wall

Large eddy simulation

Local cavity shedding

\begin{abstract}
A B S T R A C T
In this paper, the mechanisms of cavitating flow around an axisymmetric projectile near a wall with local cloud cavity shedding are analyzed using experimental and numerical methods. Several experiments are designed to observe the evolution of cavity around an axisymmetric projectile near a wall underwater with cavitation number $\sigma=0.45$. Numerical simulations using the large eddy simulation (LES) approach, Kunz cavitation model, and volume of fluid (VOF) method are established in an open-source code OpenFOAM framework to present more information on the flow structure. The shape and length of the cavity are in good agreement with the experimental observation, which guarantees the accuracy of the numerical methods. The characteristics of cavitation around the axisymmetric projectile near the wall are reported, and the wall effect is briefly analyzed according to the experimental observations and the details obtained from the numerical results. Local cavity shedding, which increases the instability of cavity periodic shedding, is observed to be induced by the combination of the vortex structure, jet at the cavity closure, and special cavity shape on the projectile near the wall.
\end{abstract}

\section{Introduction}

Cavitation is a classic issue in the hydrodynamic field that has been a subject of study in the past decades. It can be defined as the breakdown of a liquid medium under a low pressure (Franc and Michel, 2004) and widely appears on the surface of underwater vehicles, such as propellers, hydrofoils, and high-speed torpedoes. Cavitation can induce abnormal dynamic behavior, noise, and erosion that can seriously affect cloud cavitation (Soyama et al., 1992; Seo et al., 2008). Thus, the mechanisms of unsteady cavitation have been investigated from various aspects using experimental and numerical methods in the recent years.

Experiment is the main research approach for unsteady cloud cavity. For example, Kubota et al. (1989) provided a detailed description of the flow structure around unsteady cloud cavitation on a stationary two-dimensional hydrofoil using experimental methods. They showed that shed cloud is a large-scale vortex structure containing various small cavitation bubbles. Kawanami et al. (1997) investi- gated the generation mechanism of cloud cavitation in details. Callenaere et al. (2001) investigated the instability of a partial cavity induced by the development of a re-entrant jet using experiments. In the investigation of Ganesh (2015), a propagating condensation shock wave was the dominant mechanism of periodically shedding cavity. Chen et al. (2015) investigated the cavitation evolution in a convergent-divergent channel with pressure fluctuation through a tunnel experiment and numerical method. Wang et al. (2015a, 2015b) investigated the characteristics of cavity on an axisymmetric projectile near the free surface using a launching experiment and numerical method.

Numerical simulation method presents more details effectively for the clear analysis of unsteady cavitation mechanisms. Developed numerical simulation methods are based on Reynolds-averaged Navier-Stokes (RANS) equations. For example, Watanabe et al. (2003) simulated the unsteady cavitation on a propeller based on a RANS turbulence model and the Singhal cavitation model with the use of the commercial software FLUENT. The cavity shape and pressure

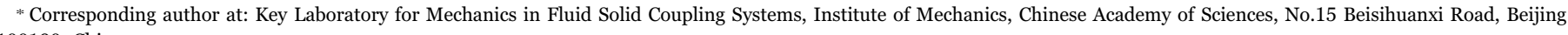
100190, China.

E-mail addresses: yuchaohust@163.com (C. Yu), wangyw@imech.ac.cn (Y. Wang), huangcg@imech.ac.cn (C. Huang), dutezhuan@imech.ac.cn (T. Du), xuchang@imech.ac.cn (C. Xu), huangjian@imech.ac.cn (J. Huang). 


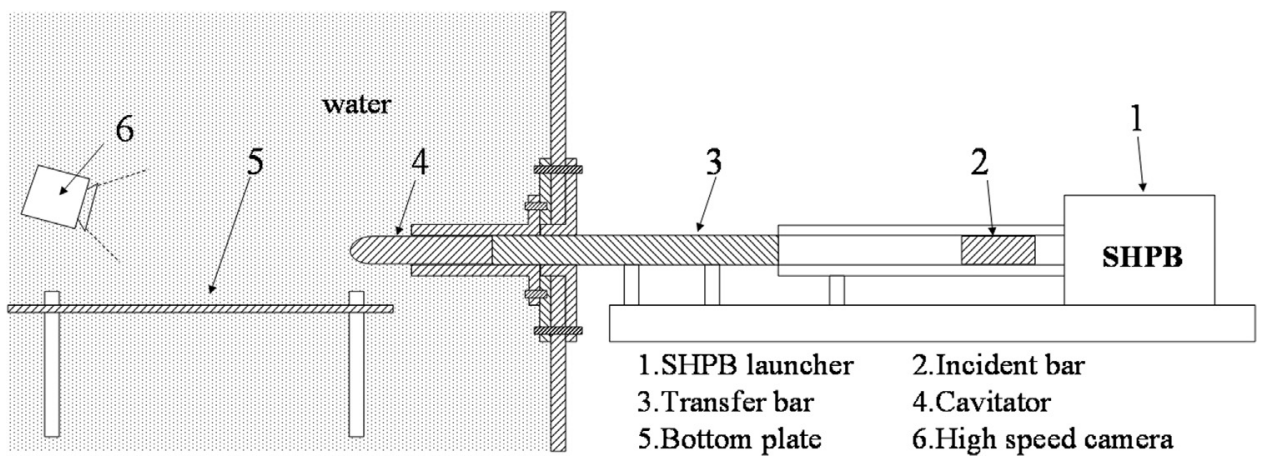

Fig. 1. Underwater launch system. (The height of bottom plate is adjustable.).

fluctuations predicted on the blade surfaces were fairly consistent with the obtained measurements. Zhou and Wang (2008) used the standard renormalization group (RNG) $k-\varepsilon$ turbulence model for the stable cavities and the modified RNG k- $\varepsilon$ model for the unstable cavity shedding. The relation between the numerical and experimental results were presented. Furthermore, RANS turbulence models have been widely used in the numerical simulations of cavitation flows around other underwater vehicles (Hasuike et al., 2009; Ji et al., 2011; Ji et al., 2012; Ying and Lu, 2008; Decaix and Goncalves, 2013; Goncalves, 2011; Wang et al., 2014).

Although RANS turbulence models are able to provide information on turbulent movement, they still have limitations when simulating the effect of transient cavitation pulsation; whereas large-eddy simulation (LES) methods have performed better in this case. Recently, many cavitating studies have adopted LES methods. For example, Bensow and Bark (2010) simulated unsteady cavitating flows around an INSEAN E779A propeller using implicit LES methods. They proved the validity of the method, pointed out that the LES of cavitation requires further development and exploration, and predicted some important cavitation mechanisms, which were useful in assessing cavitation erosion. Lu et al. (2014) simulated the cavitating flow around two highly skewed propellers operating in open water and mounted on an inclined shaft using and approach based on LES methods. Yu et al. (2014) simulated the cavitating flow around an axisymmetric projectile with cavitation number $\sigma=0.58$ using LES methods. Their numerical results were in good agreement with the experiment, and presented various cavitation details and mechanisms. Moreover, some favorable results have also been published (Dittakavi et al., 2010; Huang et al., 2014; Ji et al., 2013, 2015; Roohi et al., 2013; Wang and Ostoja-Starzewski, 2007; Wang et al., 2016a, 2016b). Besides, some new approaches are developed to simulate the cavitating flow and get favorable results (Ma et al., 2010; Ma et al., 2017).

Boundary condition, including wall effect, is an important influencing factor for cavity evolution. Studies on the effect of walls in cavitating flow are limited. For example, Xin et al. (2008) used a numerical approach to study the wall effect on ventilated cavity shape and hydrodynamics. They found that the cavity size and the drag coefficient of the ventilated cavitating flow increased with the decrease in diameter of the water tunnel, and the cavity size can be different for the same ventilation rates. In the investigation of Zhou et al. (2010) the blocking effect of water tunnel affected the ventilated super cavity shape seriously. The length of the super cavity increased with the extent of the blocking effect, while its diameter decreased. He et al. (2014) simulated the flow around a hydrofoil with various distances and showed it has significant three-dimensional characteristics because of the side wall. However, in previous literature the effect of the wall were investigated with ventilated cavity rather than natural cavitation. What's more, those investigations of the blocking effect in water tunnel mainly focused on the pressure coefficient of vehicles or volume and cycle of cavity as a whole, but rarely focused on the partial blocking effects (such as local shedding).
In this present paper, new characteristics on the cavity around the projectile near the wall are observed and analyzed in the experiment for a better understanding of the internal mechanisms of unsteady cavitation. Numerical simulations using LES approach, Kunz cavitation model (Kunz et al., 2000), and VOF method are adopted to present more details and mechanisms at a typical condition where the wall is close to the projectile. The evolution process and characteristics of cavitation around the axisymmetric projectile near the wall presented using numerical simulation are in good agreement with the experimental results. Finally, the mechanisms of the wall effect, especially on local cavity shedding, are analyzed according to the experimental observations and the details obtained from the simulations.

\section{Experimental setup}

\subsection{Experimental device}

A split Hopkinson pressure bar (SHPB) launching system is established as an experimental method to investigate the characteristics of cavitation around an axisymmetric projectile near the wall (see Fig. 1). The projectile (4) is transiently accelerated by the SHPB launching system $(1,2$, and 3$)$ with slight disturbance on the water. The distance between the projectile and the wall are changed by adjusting the height of the bottom plate (5). The high-speed camera (Phantom ${ }^{\circledast}$ v2512) (6) is used to capture the trajectory and cavitation.

\subsection{Projectile model and experiment condition}

In this study, the test model is an axisymmetric projectile with a cone head (see Fig. 2). The diameter of the projectile is $D=37 \mathrm{~mm}$ and the length is $200 \mathrm{~mm}$. The distances between the projectile and the bottom plate ( $d$, as shown in Fig. 2) are $5 \mathrm{~mm}$ to $40 \mathrm{~mm}$ in increments of $5 \mathrm{~mm}$. A contrast experiment without the bottom plate is also set up to analyze the characteristics of the cavitation around the projectile near the wall. The launch velocity is controlled by the pressure in the SHPB launching system, which is $1.2 \mathrm{MPa}$. The time-averaged velocity of the projectile is about $21 \mathrm{~m} / \mathrm{s}$ over the first $20 \mathrm{~ms}$ after launching (interferences of the velocity instability are introduced in the Appendix section). The density of water is $998.0 \mathrm{~kg} / \mathrm{m}^{3}$, the kinetic viscosity coefficient of water is $\nu=9 \times 10^{-7} \mathrm{~m}^{2} / \mathrm{s}$, and the saturated vapor pressure is $P_{\text {sat }}=3160 P_{a}$ at $25^{\circ} \mathrm{C}$. Therefore, the Reynolds number is $\operatorname{Re}=\frac{U L}{\nu}=7.77 \times 10^{5}$ (where the characteristic length is defined as the diameter of the projectile, $L=D, U$ is the time-averaged velocity of the projectile), and the cavitation number is $\sigma=\frac{P_{\infty}-P_{s a t}}{0.5 \rho_{l} U^{2}}=0.45$ (where $P_{\infty}$ is the operating pressure which is $1 \mathrm{~atm}$ at here, $\rho_{l}$ is the density of water, $U$ is the time-averaged velocity of the projectile).

\subsection{Observation in experiments}

The cavity length and the position of the projectile are obtained by measuring the pixels in the pictures. For example, the projectile with a 


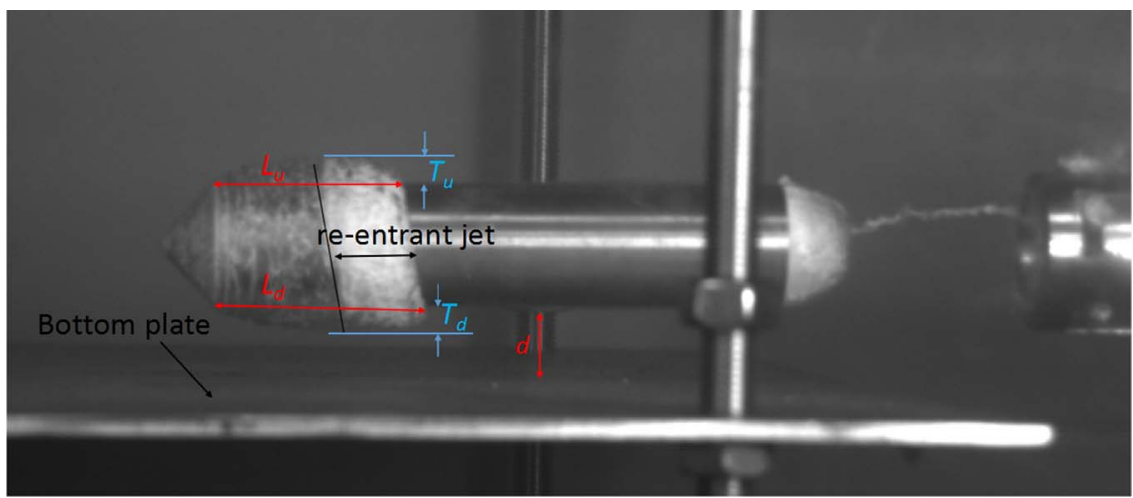

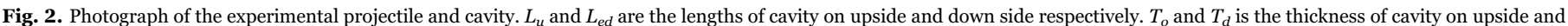
down side respectively. $d$ is the distance between the bottom plate and the down side of the projectile. The front of the re-entrant jet is marked by the black line.

length of $200 \mathrm{~mm}$ is about 408 pixels in the pictures in the experimental results. Therefore, 1 pixel is $0.49 \mathrm{~mm}$. The velocity can be calculated from the position of the projectile in adjacent frames by using the frame rate of the high-speed camera which is $16,000 \mathrm{~Hz}$.

\section{Numerical method}

A typical condition $d=15 \mathrm{~mm}$ is numerically simulated by an approach using LES methods, the Kunz cavitation model, and the VOF method to present more details and mechanisms.

\subsection{Governing equation and cavitation model}

The cavitating flow around the projectile has two phases: liquid and vapor. The VOF approach is considered to describe them with $\alpha$, introducing the liquid volume fraction (Passandideh-Fard and Roohi, 2008). In this approach, mixture viscosity $\mu$ and mixture density $\rho$ are defined as follows:

$\mu=\alpha \mu_{l}+(1-\alpha) \mu_{v}$

$\rho=\alpha \rho_{l}+(1-\alpha) \rho_{v}$

where $\mu_{l}$ and $\mu_{v}$ are the viscosities and $\rho_{l}$ and $\rho_{v}$ are the densities of liquid and vapor, respectively.

The incompressible Navier-Stokes equations of the mixture flow become:

$\nabla \bullet \vec{v}=\left(\frac{1}{\rho_{l}}-\frac{1}{\rho_{v}}\right) \dot{m}$

$\frac{\partial}{\partial \mathrm{t}}(\rho \vec{v})+\nabla \bullet(\rho \vec{v} \vec{v})=-\nabla p+\nabla \bullet \mathbf{S}$

$\mathbf{S}=2 \mu \mathbf{D}$

where $\dot{m}$ is the mass transfer rate and $\mathbf{D}=\frac{1}{2}\left(\nabla \vec{v}+\nabla \vec{v}^{T}\right)$ is the strain rate tensor.

Turbulent flow is simulated by a LES method (Yu et al., 2014; Wang et al., 2016a, 2016b; Wu et al., 2016). In the LES method, large-scale eddies are simulated by solving instantaneous Navier-Stokes equations directly, while small-scale eddies are reflected by the sub-grid scale (SGS) model (Nicoud and Ducros, 1999). The SGS model and the filter function used to distinguish large-scale from small-scale eddies are the two key components in the LES method. The spatial filtering averaging methods are as follows:

$\bar{u}_{i}(x)=\int G\left(\left|x-x^{\prime}\right|\right) u_{i}(x) d x^{\prime}$

where $G\left(\left|x-x^{\prime}\right|\right)$ is a filter function and a local function. The following box filter function is used in this study.
$G\left(\left|x-x^{\prime}\right|\right)=\left\{\begin{array}{l}1 / \sqrt[3]{\left(\Delta_{x} \Delta_{y} \Delta_{z}\right)},\left|x_{j}-x^{\prime}{ }_{j}\right| \leq \frac{\Delta_{j}}{2}, j=x, y, z \\ 0,\left|x_{j}-x^{\prime}{ }_{j}\right|>\Delta_{j} / 2\end{array}\right.$

where $\Delta_{j}$ is the spatial filter size in direction $\mathrm{j}$, which is determined by the size of grid. In this case, the thickness of the first cell adjacent to the projectile surface is $0.004 \mathrm{~mm}$ and the grid size in other directions are less than $0.4 \mathrm{~mm}$ in the vicinity of projectile.

When the filter function is applied to Eq. (3), the following LES governing equations can be obtained:

$\nabla \bullet \overline{\vec{v}}=\left(\frac{1}{\rho_{l}}-\frac{1}{\rho_{v}}\right) \dot{m}$

$\frac{\partial}{\partial \mathrm{t}}(\rho \overline{\vec{v}})+\nabla \bullet(\rho \overline{\vec{v}} \overline{\vec{v}})=-\nabla \bar{p}+\nabla \bullet(\overline{\mathbf{S}}-\mathbf{B})$

where $\mathbf{B}=\rho(\overrightarrow{\vec{v} \vec{v}}-\overrightarrow{\vec{v}} \vec{v})$ represents the influence of the small-scale eddies or the sub-grid stress tensor. The over bar denotes the filtered physical quantity.

A sub-grid viscosity $\mu_{s g s}$ is considered to solve the sub-grid stress tensor according to the Boussinesq hypothesis (Schmitt, 2007). Therefore, the sub-grid stress tensor becomes:

$\mathbf{B}=-2 \mu_{s g s} \overline{\mathbf{D}}$

The sub-grid viscosity $\mu_{s g s}$ is solved using the $k-\mu$ model:

$\frac{\partial k_{s g s}}{\partial t}+\nabla \cdot\left(k_{s g s} \bar{v}\right)=\nabla \cdot\left[\frac{\mu+\mu_{s g s}}{\rho} \nabla k_{s g s}\right]+2 \frac{\mu_{s g s}}{\rho} \bar{D} \bar{D}-C_{e} \frac{k_{s g s}^{3 / 2}}{\bar{\Delta}}$

$\mu_{s g s}=C_{k} \bar{\Delta} \sqrt{k_{s g s}}$

where $k_{s g s}$ is the SGS turbulent kinetic energy, $\bar{\Delta}=\sqrt[3]{\Delta_{x} \Delta_{y} \Delta_{z}}$ is the average spatial filter size, $C_{e}=1.048$ and $C_{k}=0.094$.

In the unsteady cavitation flow, the transition between liquid and vapor is complex. The liquid volume fraction solved by the VOF approach with a transport equation as:

$\frac{\partial \alpha}{\partial t}+\nabla \bullet(\overline{\vec{v}} \alpha)=\frac{\dot{m}}{\rho_{v}}$

where $\dot{m}$, the mass transfer rate is solved by the Kunz cavitation model (Kunz et al., 2000):

$\dot{m}^{+}=\frac{C_{v} \rho_{v} \alpha \min \left[0, \bar{p}-p_{v}\right]}{\left(1 / 2 \rho_{l} U_{\infty}^{2}\right) t_{\infty}}$

$\dot{m}^{-}=\frac{C_{c} \rho_{v} \alpha^{2}(1-\alpha)}{t_{\infty}}$

where $p_{v}$ is the saturated vapor pressure of water, $\dot{m}^{+}$is the evaporation rate, and $\dot{m}^{-}$is the condensation rate. In Eq. (11), $U_{\infty}$ is the speed of projectile. The characteristic time $\left(t_{\infty}\right)$ is equal to $D / U_{\infty}$, where $D$ is the diameter of the projectile. The empirical constants for the different 


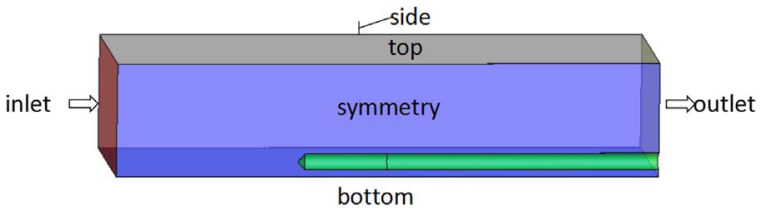

Fig. 3. Computational model and domain.

phase transfer rates are denoted by $C_{v}$ and $C_{c}$. The empirical constants are set as: $C_{v}=200$ and $C_{c}=100$, which are chosen from vast computational experience (Wang et al., 2014, 2015a, 2015b; Yu et al., 2014, 2017).

\subsection{Numerical procedures}

Similar with the experimental projectile, a cylinder with a cone head is adopted in the numerical simulation. As the experimental conditions and phenomenon are symmetrical in the horizontal direction, physical quantities (such as pressure, velocity and volume fraction of vapor) should be symmetrical in this direction approximately. Thus, only half of the horizontal direction of the hydrofoil is considered to reduce calculation cost, using a symmetry plane at the center of the projectile in our computational experience (Lu et al., 2010; Wang et al., 2016a, 2016b; Wu et al., 2016). The computation domain includes half of the cylinder, and the cross-section is set as a symmetry plane (see Fig. 3). The inlet plane is $400 \mathrm{~mm}$ away from the cylinder head, and the outlet plane is $800 \mathrm{~mm}$ away from the cylinder head. The top plane is $200 \mathrm{~mm}$ away from the up side of the cylinder, and the side boundary is $400 \mathrm{~mm}$ away from the cylinder. The distance between the bottom boundary and the down side of the cylinder is determined by $d$. A typical condition, $d=15 \mathrm{~mm}$, is simulated.

The inlet boundary is a velocity-inlet with a fixed velocity of $21 \mathrm{~m} / \mathrm{s}$. The outlet and top boundary are a pressure-outlet with a static pressure of $101325 \mathrm{P}_{\mathrm{a}}$. The side and bottom boundary are a moving wall with a velocity of $21 \mathrm{~m} / \mathrm{s}$ in the $\mathrm{x}$-direction.

A block-structured grid in the computation domain is prepared (see Fig. 4). From Section 2.2, $\operatorname{Re}=\frac{U L}{\nu}=7.77 \times 10^{5}$. At such a high Reynolds number, LES cannot resolve eddies in the semi-viscous near-wall region, unless a very fine mesh is used. Even if such a fine mesh could be accommodated normal to the wall, the reduction in the turbulent scale in all three directions implies the need for similar refinements in the other two directions. This is not tenable on economic grounds and necessitates the adoption of an approximate treatment which bridges the near-wall layer (Temmerman et al., 2003). Thus, on the surface of the projectile, a wall model (De Villiers, 2007) is used and the thickness of the first cell adjacent to the projectile surface is $0.004 \mathrm{~mm}$ (about $D / 10^{5}$ ) which satisfied $y^{+}=\rho_{l} u_{\tau} y / \mu$ (where $y$ is the thickness of the first cell adjacent to the projectile surface) value less than 10 . For the

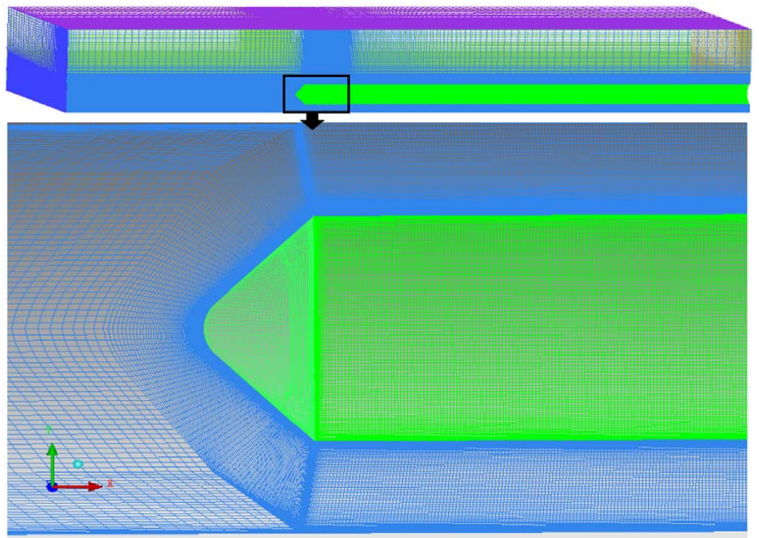

Fig. 4. Computational mesh. surface mesh, the grid sizes are less than $0.4 \mathrm{~mm}$ in the vicinity of the shoulder of projectile. This near wall resolution is deemed sufficient for reliable wall-modeled LES based on our experiences with the applied wall model.

The cavity shapes between the simulation results of coarse and middle size grids have many differences, specifically the cavity shape on the up side of the projectile at $t=7 \mathrm{~ms}$ (see Fig. 5). The cavity shapes and evolution are similar in the simulation results of middle size and fine grids. Therefore, the middle size grid with 5 million cells is selected as the final grid.

\subsection{Simulation procedures}

As proposed by Yu et al. (2014) and Wu et al. (2016), the numerical simulation is realized using the OpenFOAM framework with the firstorder Euler implicit scheme for time discretization and Gauss linear scheme for spatial discretization. An adjustable time step is used with maximum Courant number (Co) of 0.3 to reach a high-accuracy in time. The precise time steps are less than $1 \times 10^{-6} \mathrm{~s}$. The computational time is approximately $80 \mathrm{~h}$ on 128 Intel Xeon E5620, $2.40 \mathrm{GHz}$ CPU cores.

\section{Results and discussion}

\subsection{Comparison of experimental and numerical results}

Evolution of the cavity shapes, the length of the cavity on the up side $\left(L_{u}\right)$ and down side $\left(L_{d}\right)\left(L_{u}\right.$ and $L_{d}$ are shown in Fig. 2) obtained from the numerical simulation are compared with the experimental observations.

The incipient cavitation appears at the shoulder of the projectile. The cavity bubble has a growth stage after launching. In this stage, the length of the cavity increases with a speed close to the launching speed of the projectile as shown in Fig. 7, Stage 1-1. Because the relative speed between the projectile and water is $21 \mathrm{~m} / \mathrm{s}$ in the initial field, the length of sheet cavity before the shoulder of projectile increase with the maximum flow speed along the cavity length about $21 \mathrm{~m} / \mathrm{s}$. The effect of the near plate on the length of cavity start to become significant when the growth of cavity length slow down. Thus, there is little difference between the shapes of the sheet cavity on the up and down side, as shown in Fig. 6(a).

After the growth stage, a re-entry jet developing stage is shown in Fig. 6(b-d) and the variation of cavity length is shown in Fig. 7(Stage 1-2). Then the cavity sheds as shown in Fig. 6(e, f) and the variation of cavity length in this stage is shown in Fig. 7(stage 2-1). After shedding, the cavity collapses as shown in Fig. $6(\mathrm{~g})$ and the re-entry jet is also generated for the second time as shown in Fig. 6(h). In this stage, the variation of cavity length is shown in Fig. 7(stage 2-2).

In the experimental and numerical results, a significant difference between the lengths of the cavity on the up and down sides of the projectile in stages 1-2 and 2-1 exists (see Fig. 7). In stage 1-2, the $L_{d}$ fluctuates apparently because a small-scale cloud cavitation sheds at $t=$ $7 \mathrm{~ms}$ and moves toward the wall on the down side of the projectile subsequently (Fig. 6(c, d)).

From the analysis above, the numerical simulations obtain the key characteristics observed in the experiments. The lengths of $L_{u}$ and $L_{d}$ are predicted in relation to the experimental results. Several differences are evident after the primary cavity shedding, which are probably caused by the decreases in the velocity and height of the projectile after launching (more details of the interferences can be found in the Appendix).

\subsection{Characteristics of cavity under the near wall effect}

When the projectile is launched without the boundary effect, the cavity around the projectile is periodic and axisymmetric. Detailed 


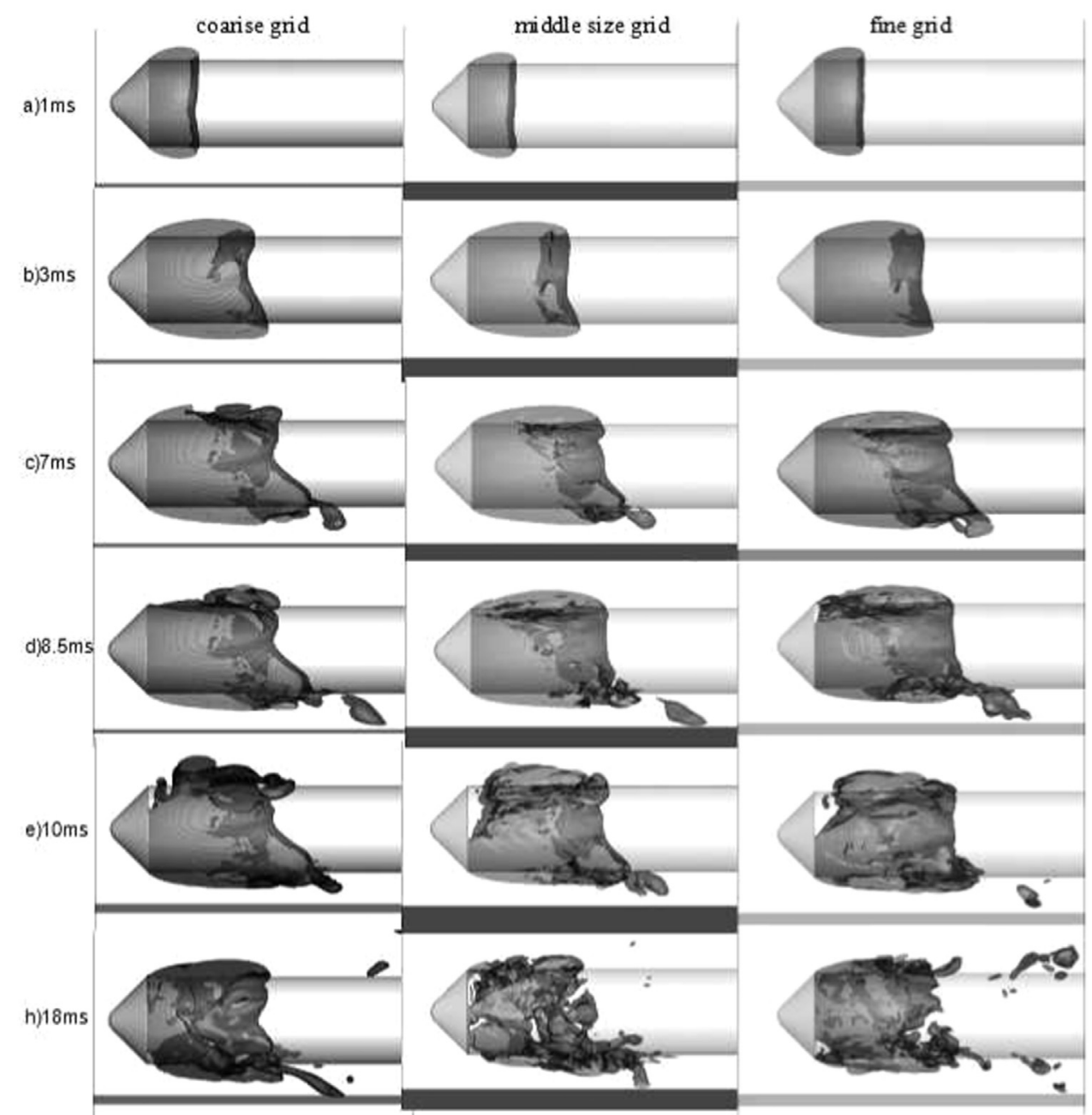

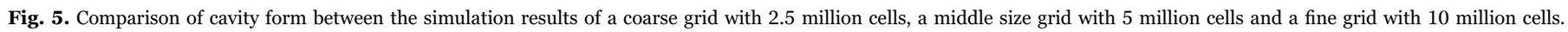

experimental results and analysis can be found in previous literature (Yu et al., 2014). When the projectile is launched near the wall, the cavitation around the axisymmetric projectile has many non-axisymmetric characteristics and is more unstable. Based on the experimental observations, when $d$ is less than $35 \mathrm{~mm}$, the wall has evident effects on the shape and evolution of the cavity on the side near the wall. The characteristics of cavitation around the axisymmetric projectile near the wall can be described through the typical condition $d=15 \mathrm{~mm}$ as follows.

At the end of the growth stage, the cavity length is relatively long on the side near the bottom plate (Figs. 6(b) and 7). Then, the front end of the re-entry jet in the down side runs behind the up side in the re-entry jet developing stage (Fig. 6(c) and Fig. 7). On the side near the bottom plate, the primary cavity sheds behind the other side, which increases the instability for the periodic cavity shedding (see Fig. 6(d, e)). When $d$ is small enough, the periodic cavity shedding becomes more unstable because a local cavity shedding appears at the cavity closure on the side near the bottom plate in the re-entry jet developing stage (Fig. 6c, d, and e). Furthermore, the thickness of the cavity on the down side is smaller than that at the up side (see Fig. 8).

\subsection{Analysis of the wall effect}

According to the experimental observations and the details obtained from the simulations, the mechanisms of the wall effect can be briefly analyzed.

In the growth stage, the flow is relatively steady. Many differences exist between the stream lines on the down side and up side at the region around the cone head because of the blocking effect of the bottom plate as well as the pressure distribution (Fig. 9). A relatively high pressure also exists on the cavity closure. The axial velocity near the down side of the projectile is larger than that at the up side (Fig. 10). From the Bernoulli equation, the pressure on the region near the down side of the projectile is relatively lower than that at the up side. Thus, the low pressure region on the down side is larger than the up side as shown in Fig. 9, and the cavity length on the down side is longer than that on the up side. This also makes the growth stage of the cavity on the down side longer than that on the up side. At the end of the growth stage, a vortex appears on the end of the cavity (as shown in Fig. 9), which is also reflected by the flow separation on the upper side of the projectile (as shown in Fig. 10).

Furthermore, the angle between the projectile surface and the velocity of the flow on the down side becomes smaller than that on the up side because of the blocking effect of the bottom plate (Fig. 9). This partly makes the cavity thickness on the down side smaller than that on the up side. When the distance between the projectile and bottom plate becomes even smaller, the space limits the development of cavity thickness on the down side ( $T_{d}$ is definitely smaller than $d$, as shown in Fig. 2).

The re-entry jet on the down side develops behind the up side because the growth stage of the cavity on the down side is longer than that on the up side. This partly makes the front end of the re-entry jet in the down side run behind the up side. At the end of the re-entry jet developing stage, the re-entry jet arrives at the shoulder on the up side 

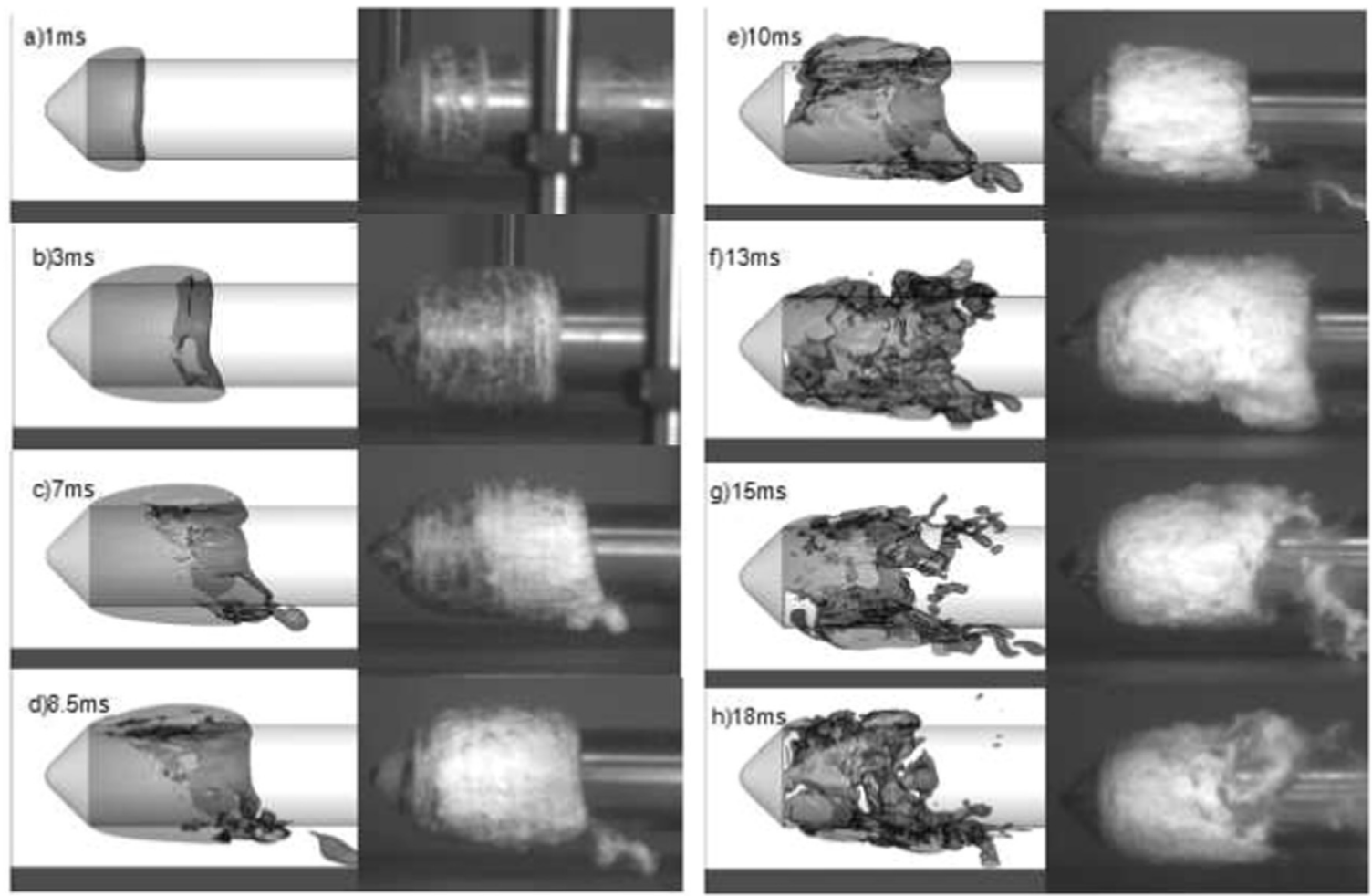

Fig. 6. Evolutions of cavity when $d=15 \mathrm{~mm}$. Cavities on the left are numerical results (reflected by the isosurface of $\alpha=0.9$ ), on the right are experimental results.

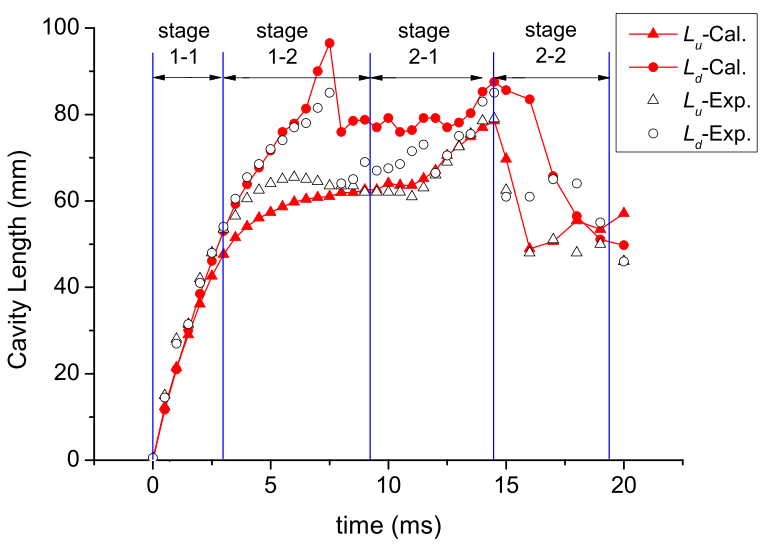

Fig. 7. Evolution of cavity length when $d=15 \mathrm{~mm}$. $L_{u}$-Cal. and $L_{u}$-Exp. are the numerical and experimental length of cavity on the down side respectively. $L_{u}$-Cal. and $L_{u}$-Exp. are the numerical and experimental length of cavity on the down side respectively.

and cuts the cavity on the up side before the down side (Fig. 11). Therefore, the primary cavity sheds on the down side after the up side.

\subsection{Analysis of local shedding}

The cavity on the side near the bottom plate is more unstable than the other side because of the local cavity shedding. Compared with the primary cavity shedding, the local cavity shedding is worth studying because it is one of the key characteristics of the cavitation under the wall effect.

The local shedding cloud cavitation moves with continuously in the experimental observations. In this paper, the local cavity shedding is analyzed with the vortex structure, which is represented by the $Q$ criterion (Sahner et al., 2005).

In the incompressible flow, $Q$ is defined as:
$Q=\frac{1}{2}\left(\|\Omega\|^{2}-\|D\|^{2}\right)$

where $\Omega=\frac{1}{2}\left(\nabla \vec{v}-\nabla \vec{v}^{T}\right)$ represents the vorticity tensor and $D=\frac{1}{2}\left(\nabla \vec{v}+\nabla \vec{v}^{T}\right)$ represents the strain rate tensor. $Q$ has a direct physical meaning. When $Q>0$, the vector field is dominated by the vorticity and the region is determined as a vortex tube. When $Q<0$, the vector field is dominated by the strain.

Fig. 12 shows that the shape of the vortex structures is very similar to that of the cavity, which shows a close relationship between the local shedding cavity and vortex motion. Considering the analysis of vortex motion in the evolution of local shedding using numerical simulation, the local shedding is mainly caused by the vortex motion in the end of cavity and the cavity shape, which is relatively long on the side near the wall.

In the re-entry jet developing stage, a vortex exists at the end of the cavity (see Fig. 13). The direction of the vortex is the tangential direction of the end line of the cavity. If the cavity is axisymmetric, a circular vortex ring would appear at the end of the cavity, which promotes the re-entry jet toward the shoulder of the projectile. However, the cavity is relatively longer on the down side in this case. Therefore, the re-entry jet promoted by the vortex and the high pressure behind the cavity (see Fig. 9) moves obliquely downward and converge at the down side of the projectile (see Fig. 14). When the downward component of the jet is strong enough, the jet breaks the cavity and joins the outflow of the cavity on the down side of the projectile. After breaking the cavity, the cavity behind the jet separates from the primary cavity at the end of the cavity.

After separating from the primary cavity, the small scale cloud cavity sheds downstream with the liquid flow and moves to the bottom plate in the same time. Considering the numerical results analysis, the local shedding cavity moves to the bottom plate mainly because of the vortex motion. A pair of vortex sheds with the cavity, and the mutually induced velocity of the vortex is downward (see Fig. 15). Therefore, the local shedding cavity moves toward the bottom plate with the vortex after being separated from the primary cavity. 


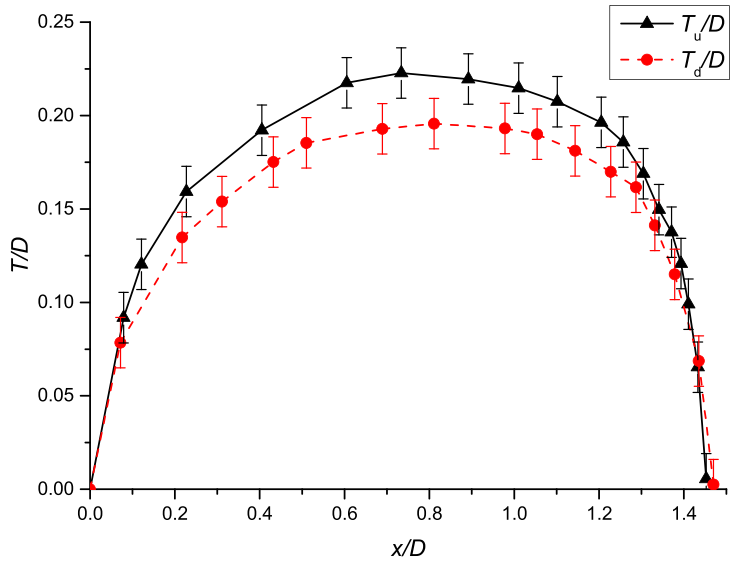

(a)

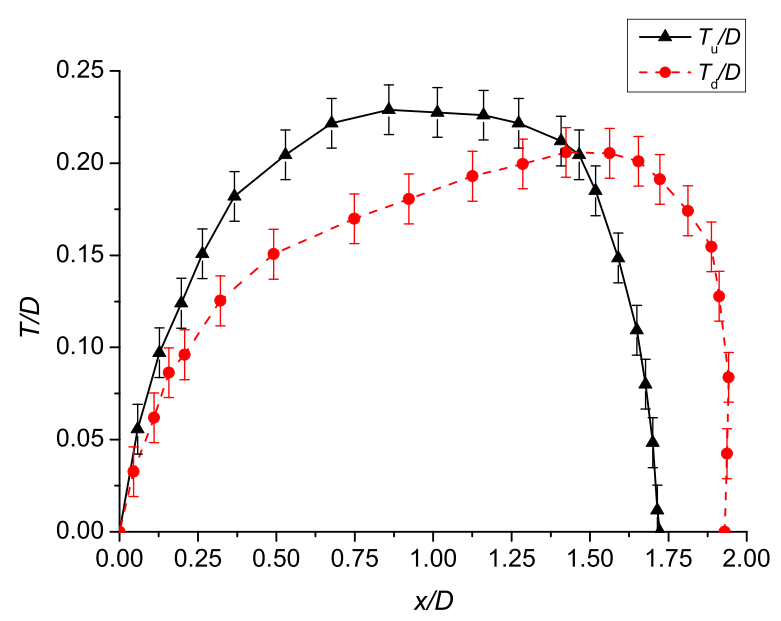

(b)

Fig. 8. The thickness of the cavity on the down side and the up side of the projectile (With $d=15 \mathrm{~mm}$, at $t=3 \mathrm{~ms}$ in figure (a), $t=5 \mathrm{~ms}$ in figure (b)).

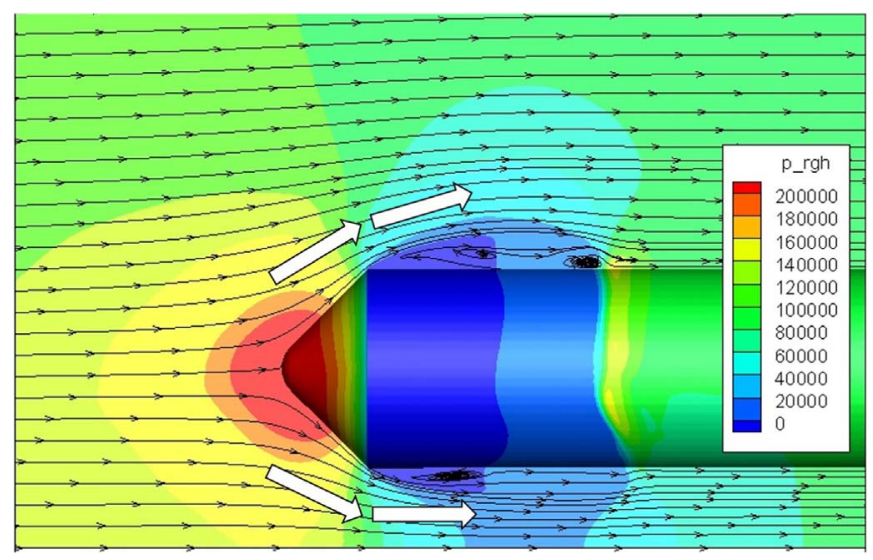

Fig. 9. Stream lines and pressure distribution on the symmetry plane at $t=3 \mathrm{~ms}$.

\subsection{Effect of the distance between the projectile and wall}

The dimensionless parameter $d / D$ (where $d$ is the distance between the bottom plate and the down side of projectile, $D=37 \mathrm{~mm}$ is the diameter) is one of the control parameter when the Cavitation number and Reynolds number are little changed. Different distances between the bottom plate and the down side of the projectile are observed in the experiment to investigate its' effect on the cavity.

When $d$ is less than $35 \mathrm{~mm}$, the length of the cavity on the down side $\left(L_{d}\right)$ is longer than the up side $\left(L_{u}\right)$ in the re-entry jet developed

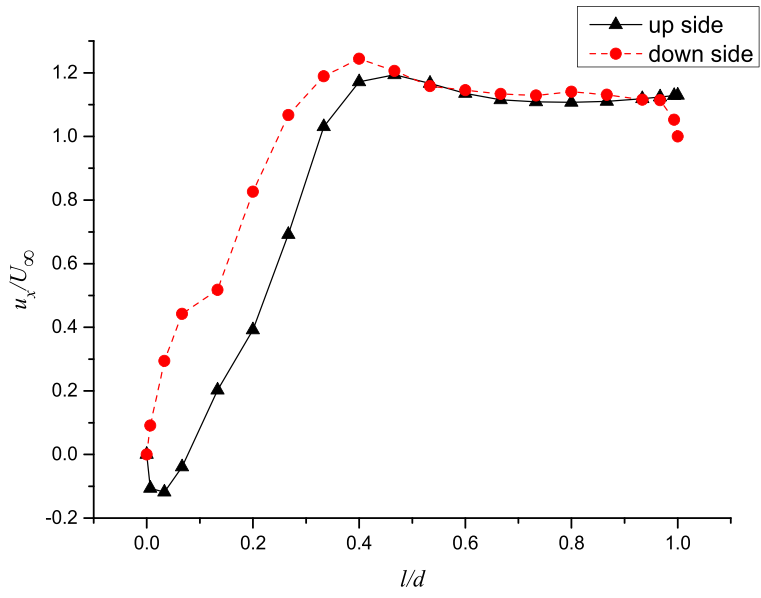

Fig. 10. Comparison of axial velocity on the up side and down side in the symmetry plane at the position of $1 D$ behind the shoulder in numerical results when $t=4 \mathrm{~ms}$. In the horizontal axis, $l$ is the distance away from the projectile, $d$ is the distance between the projectile and wall $\left(d=15 \mathrm{~mm}\right.$ at here). $U_{\infty}$ is the characteristic velocity $\left(U_{\infty}=21 \mathrm{~m} / \mathrm{s}\right), u_{x}$ is the axial velocity.

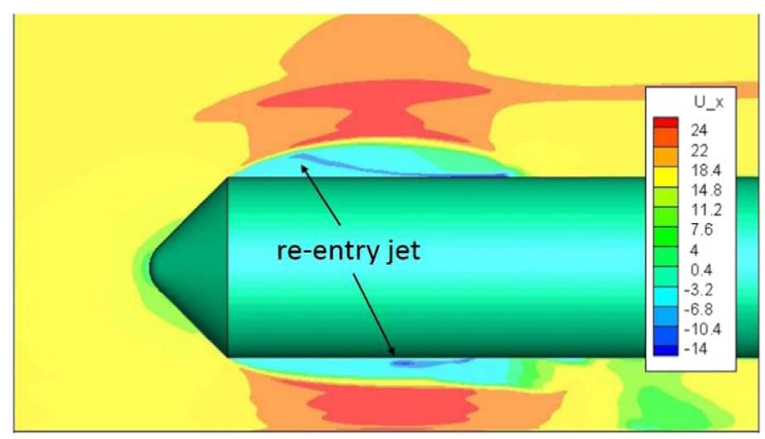

Fig. 11. Distribution of axial velocity and re-entry jet in the symmetry plane at $t=8 \mathrm{~ms}$.

stage (as shown in Fig. 16). As shown in Table 1, the difference between $L_{d}$ and $L_{u}$ becomes significant $\left(L_{u}-L_{d}\right.$ increase) with the distance from the wall $(d)$ decrease at $t=5 \mathrm{~ms}$ after launching when the re-entry jet began to develop.

When $d \leq 20 \mathrm{~mm}$, the difference between $L_{d}$ and $L_{u}$ is large enough and a small scale shedding cloud cavitation (local cavity shedding) appears at the end of cavity in the down side before the primary cavity sheds from the shoulder (Fig. 17).

When $25 \mathrm{~mm} \leq d \leq 35 \mathrm{~mm}$, the wall effect on cavity thickness is small. In the cavity growth and re-entry jet developed stages, the cavity thickness on the down side $\left(T_{d}\right)$ is approximately equal to that on the up side $\left(T_{u}\right)$ (see Table 1). However, the shedding cloud cavitation on the down side moves to the bottom plate and becomes thicker than that on the up side (Fig. 18). From Table 2, differences are small between the maximum thickness of the shedding cloud cavity on the up and down side of the projectile when $25 \mathrm{~mm} \leq d \leq 35 \mathrm{~mm}$.

When $d \leq 15 \mathrm{~mm}$, the cavity thickness on the down side $\left(T_{d}\right)$ is shorter than that on the up side $\left(T_{u}\right)$ in the cavity growth and re-entry jet developed stages (Fig. 10) and $T_{d}$ decreases with $d$, as shown in Table 1. From Table 2, the maximum thickness of the shedding cloud cavity on the down side of the projectile approximately equal to $d$ as the shedding cloud cavity almost touching the bottom plate.

\section{Conclusions}

In present paper, several experiments observing the characteristics of the cavity around the axisymmetric projectile near a wall are designed to study the wall effect on the natural cavitation around the projectile. Numerical simulations using a LES approach, the Kunz cavitation model, and the VOF method are performed to present more 


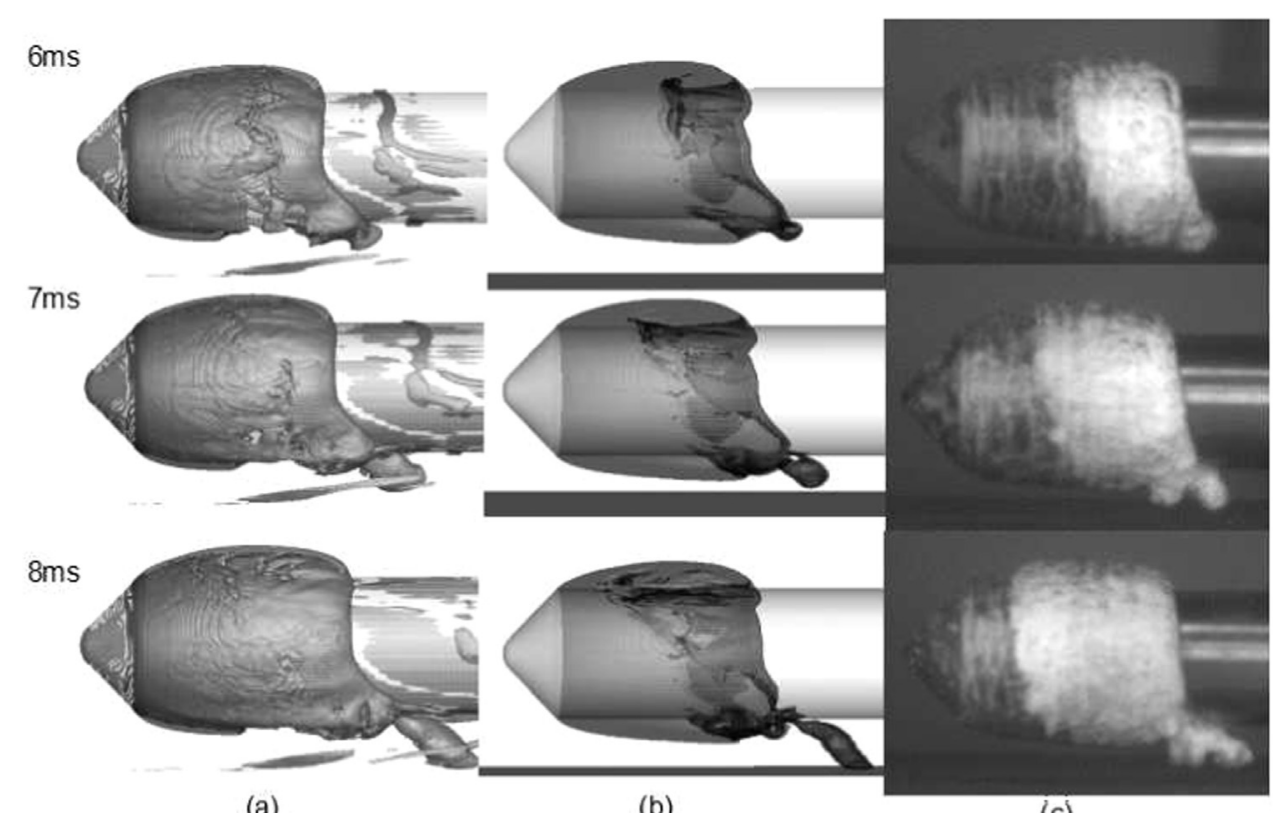

(a)

(b)

(c)

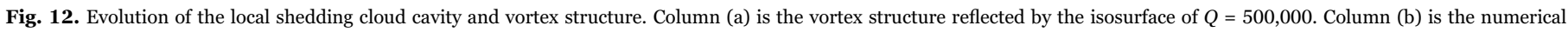
cavity which reflected by the isosurface of $\alpha=0.9$. Column (c) is the experimental cavity.

details and mechanisms at a typical condition $d=15 \mathrm{~mm}$. The evolutions of the shape and length of the cavity are presented and are in good agreement with the experimental results of this numerical method.

The experimental observations indicate that the effective distance between the projectile and bottom plate has a significant effect on the cavity shape around the axisymmetric projectile, which is determined as $d \leq 35 \mathrm{~mm}$ (or $d / D \leq 0.95$ ) when cavitation number $\sigma=0.45$. When $d$ $\leq 35 \mathrm{~mm}$, the cavity length on the up side of the projectile $\left(L_{u}\right)$ is shorter than that on the down side $\left(L_{d}\right)$ and becomes more significant with the decrease of $d$ in the cavity growth and re-entry jet developed stages. The front end of the re-entry jet in the down side runs behind the up side. When $d \leq 20 \mathrm{~mm}$ (or $d / D \leq 0.54$ ), local cavity shedding appears at the end of the cavity on the side near the wall. In addition, in the cavity growth and re-entry jet developed stages, the thickness of the cavity on the down side $\left(T_{d}\right)$ is shorter than that on the up side $\left(T_{u}\right)$ and decrease with $d$ when $d \leq 15 \mathrm{~mm}$. When $25 \mathrm{~mm} \leq d \leq 35 \mathrm{~mm}$, the primary shedding cloud cavitation moves toward the bottom plate.

Based on the experimental observations and the numerical results, the bottom plate has a blocking effect on the flow around the cone head on the down side of the projectile. Compared to the other side, the axial velocity on the side near the wall is larger, and the pressure distribution changes because of the blocking effect. Such a non-axisymmetric velocity and pressure distribution lead to the non-axisymmetric cavity shape, which is relatively longer on the side near the wall in the end of the cavity growth stage, and the characteristics in the following stages.

When launched near the bottom plate, the cavity around the projectile no longer has stable periodic shedding. On the one hand, the cycle of primary cavity shedding on the side near the wall is longer than that on the other side. On the other hand, the local cavity shedding increases the instability of cavity periodic shedding. Under the combined action of high pressure at the cavity closure and the nonaxisymmetric cavity shape in the re-entry jet developed stage, the reentry jet in left and right sides of the projectile converge at the down side and form a jet. Then the jet moves obliquely downward and tears the primary cavity on the down side and separates a small scale cloud cavity from the primary cavity. This separated cavity then sheds with the vortex at the cavity closure and moves toward the wall under the effect of this vortex motion.

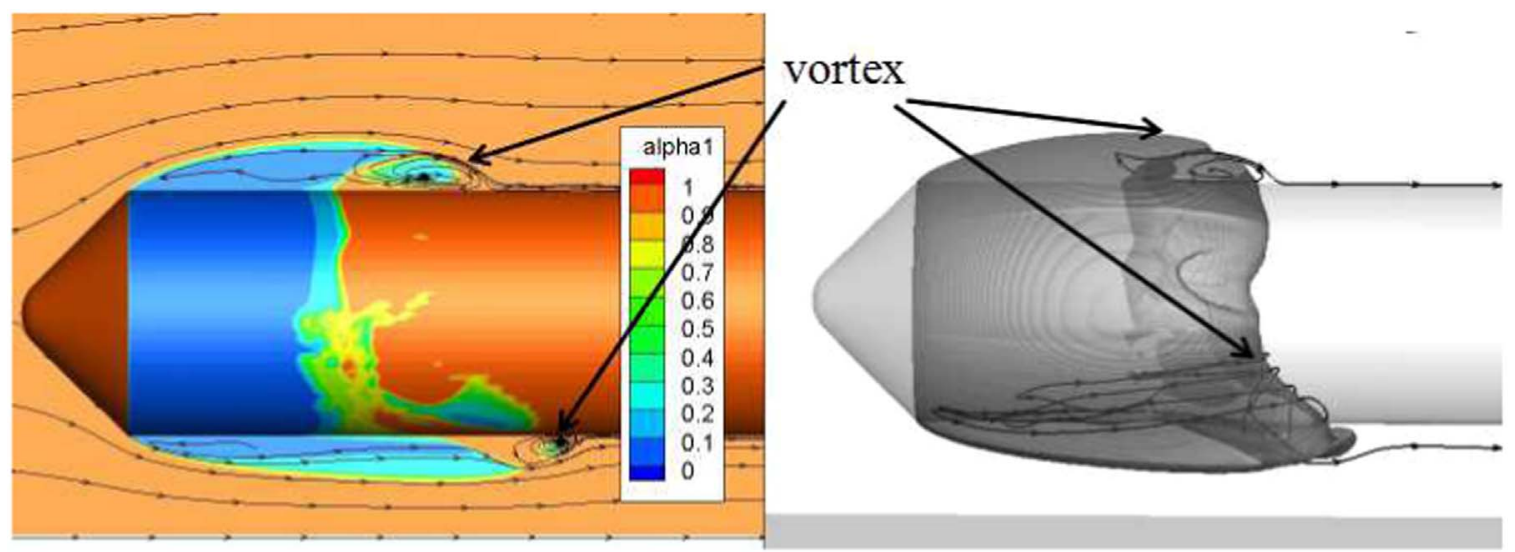

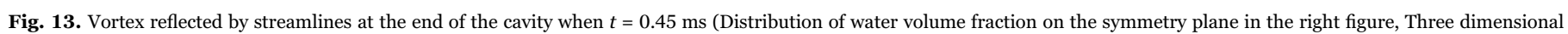
cavity reflected by the isosurface of $\alpha=0.9$ in the left figure.). 


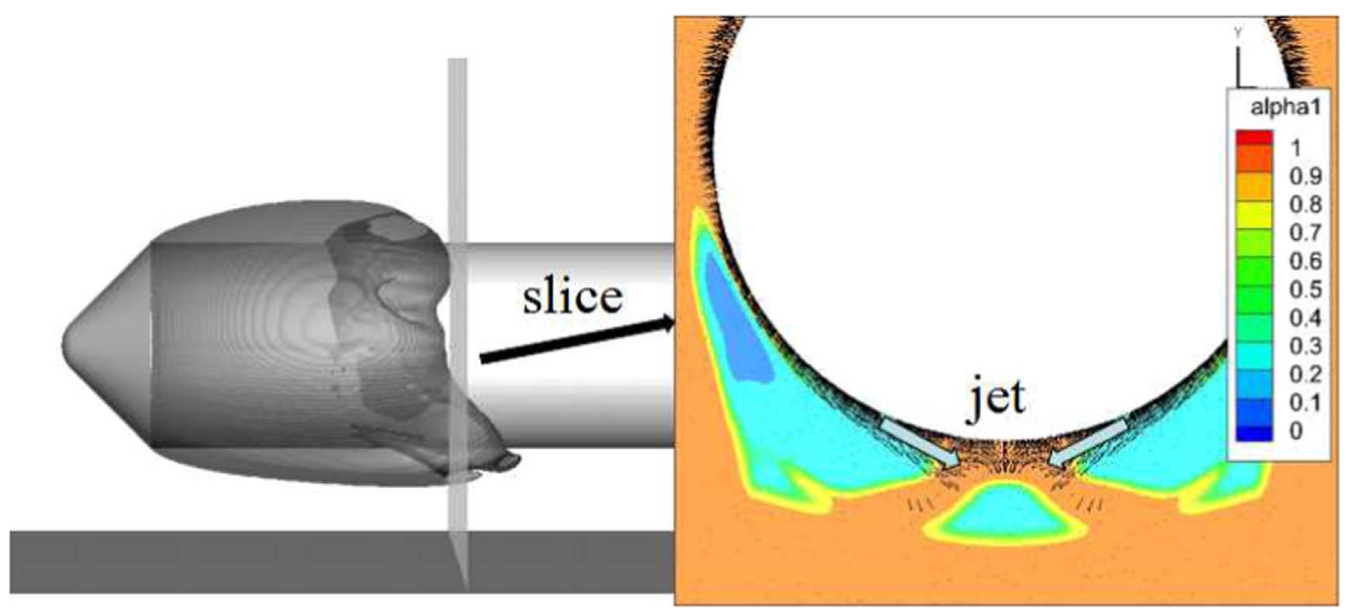

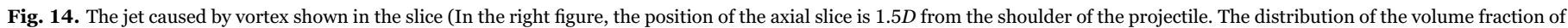
water, which named alpha1, and the surface velocity vectors on the slice are shown in the left figure. The jet is marked by the white thick vector in the left figure.).

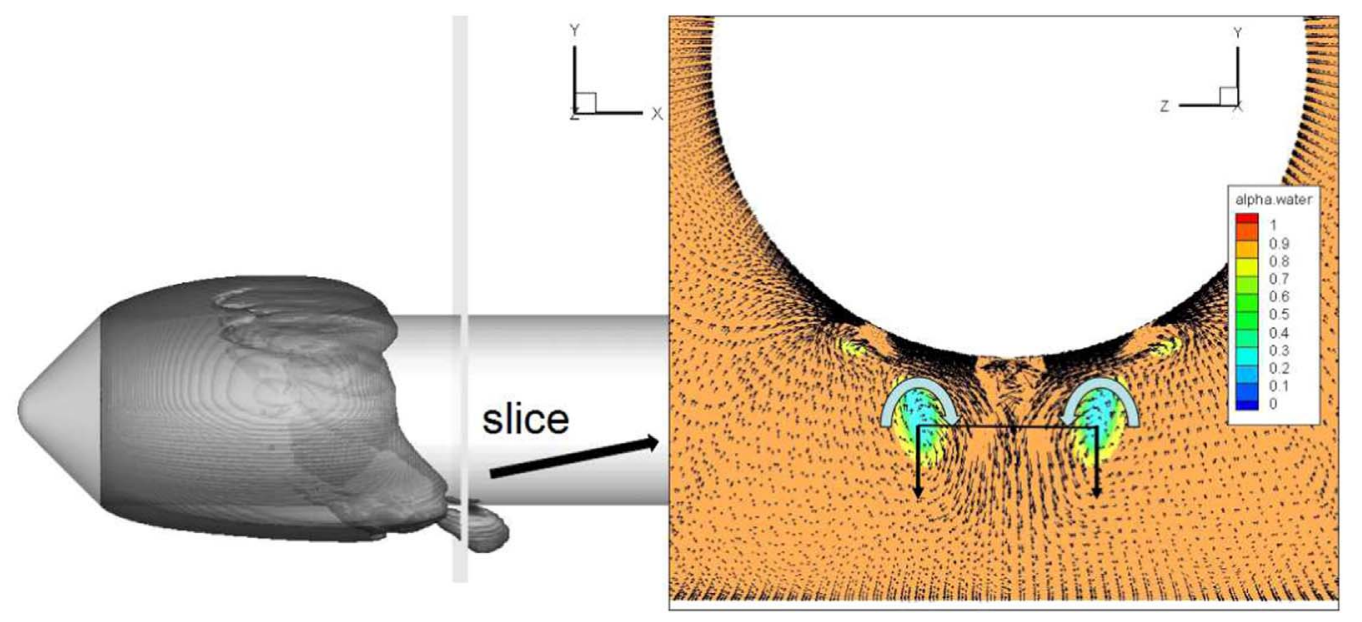

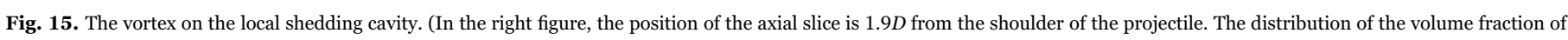

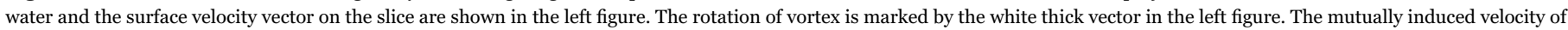
the pair of vortex is marked by the black thick vector.).

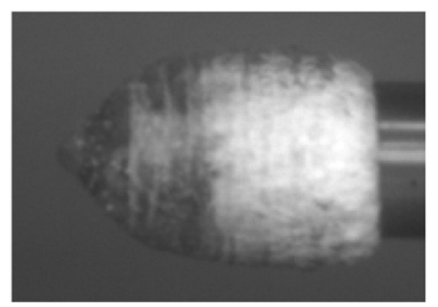

(a) $d=35 \mathrm{~mm}$

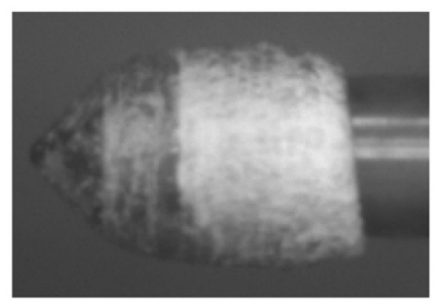

(b) $d=25 \mathrm{~mm}$

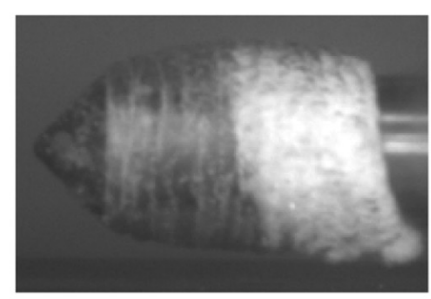

(c) $d=15 \mathrm{~mm}$

Fig. 16. Cavity and re-entry jet.

Table 1

The length of the cavity on the down side $\left(L_{d}\right)$ and the up side $\left(L_{u}\right)$ and the thickness of the cavity on the down side $\left(T_{d}\right)$ and the up side $\left(T_{u}\right)$ of the projectile at $t=5$ ms.

\begin{tabular}{|c|c|c|c|c|c|c|c|c|}
\hline$d(\mathrm{~mm})$ & 5 & 10 & 15 & 20 & 25 & 30 & 35 & 40 \\
\hline$L_{u}(\mathrm{~mm}) \pm 0.5 \mathrm{~mm}$ & 67.0 & 61.5 & 63.0 & 59.0 & 56.5 & 63.0 & 57.0 & 56.5 \\
\hline$L_{d}(\mathrm{~mm}) \pm 0.5 \mathrm{~mm}$ & 87.0 & 74.5 & 71.0 & 65.5 & 60.0 & 64.5 & 58.0 & 56.5 \\
\hline$L_{u}-L_{d}(\mathrm{~mm}) \pm 1.0 \mathrm{~mm}$ & 20 & 13 & 8 & 6.5 & 3.5 & 1.5 & 1.0 & 0 \\
\hline$T_{u}(\mathrm{~mm}) \pm 0.5 \mathrm{~mm}$ & 8.5 & 8.5 & 8.5 & 8.5 & 9.0 & 9.0 & 8.5 & 8.5 \\
\hline$T_{d}(\mathrm{~mm}) \pm 0.5 \mathrm{~mm}$ & 4.0 & 6.0 & 8.0 & 8.5 & 8.5 & 9.0 & 9.0 & 8.5 \\
\hline
\end{tabular}




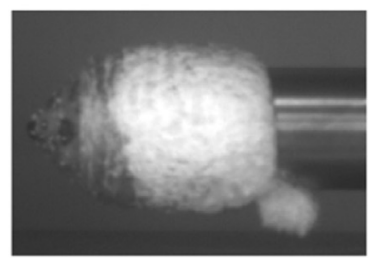

(a) $d=20 \mathrm{~mm}$

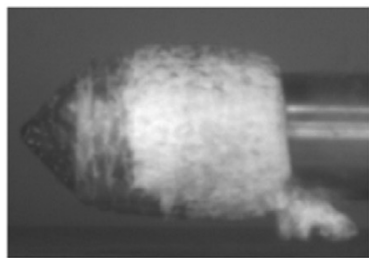

(b) $d=15 \mathrm{~mm}$

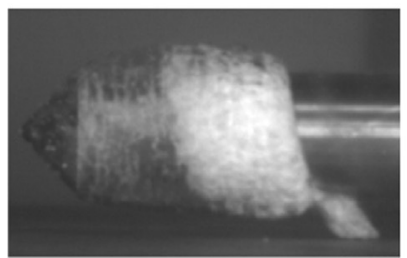

(c) $d=10 \mathrm{~mm}$

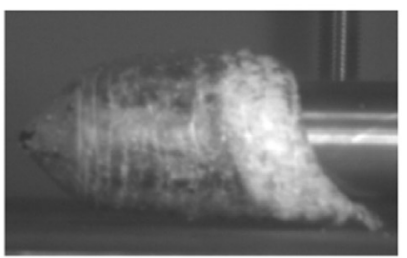

(d) $d=5 \mathrm{~mm}$

Fig.17. Local cloud cavitation shedding.

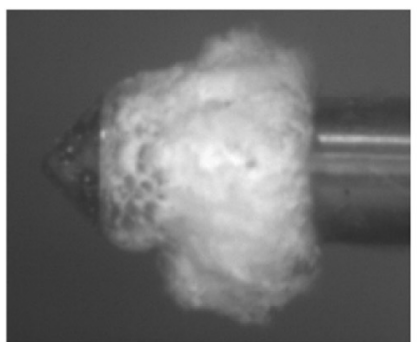

(a) $d=35 \mathrm{~mm}$

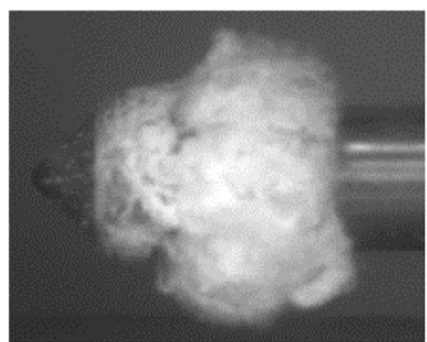

(b) $d=25 \mathrm{~mm}$

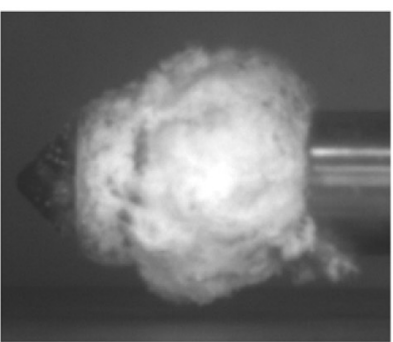

(c) $d=15 \mathrm{~mm}$

Fig.18. Primary cloud cavitation shedding.

Table 2

The maximum thickness of the shedding cloud cavity on the down side $\left(T_{d \max }\right)$ and the up side $\left(T_{u \max }\right)$ of the projectile on different conditions.

\begin{tabular}{|c|c|c|c|c|c|c|c|c|}
\hline$d(\mathrm{~mm})$ & 5 & 10 & 15 & 20 & 25 & 30 & 35 & 40 \\
\hline$T_{d \max }(\mathrm{mm}) \pm 0.5 \mathrm{~mm}$ & 18.5 & 20.0 & 19.0 & 17.0 & 21 & 20.5 & 20.5 & 17.0 \\
\hline$T_{u \max }(\mathrm{mm}) \pm 0.5 \mathrm{~mm}$ & 5.0 & 9.5 & 15.0 & 15.5 & 21 & 21.5 & 21.0 & 17.0 \\
\hline
\end{tabular}

\section{Acknowledgment}

The authors are grateful to National Natural Science Foundation of
China through grant numbers 11202215 and 11332011, and the Youth Innovation Promotion Association of CAS (2015015).

\section{Appendix. Experimental Interfering factors}

In this experiment, many interfering factors are difficult to avoid. The velocity of projectile decreases after launching because of the resistance in water. Meanwhile, the projectile body is deflected and the projectile height changes because of the gravity and the non-axisymmetric distribution of pressure when the projectile launches near the bottom plate. Besides, as shown in Fig. 2, there are 4 struts of the bottom plate in water tank. But the distances between the projectile and the struts are about $400 \mathrm{~mm}$, which is more than ten times of the projectile diameter. The effect of struts can be ignore at such distances.

In the first $20 \mathrm{~ms}$ after the projectile launches, the velocities drop approximately $7 \%$, the dip angles are less than $1.5^{\circ}$, the distance between the projectile and bottom plate changes less than $20 \%$ in experiments No. 2-8 (see Table A1). The interfering factors are slight enough to ensure the

\section{Table A1}

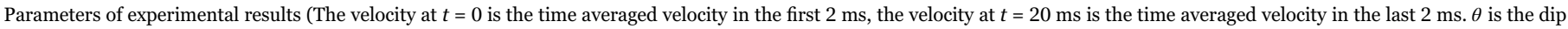
angle of the projectile, and the minus dip angle means the cone head of projectile rotating downward).

\begin{tabular}{|c|c|c|c|c|c|c|}
\hline \multirow[t]{2}{*}{ No. } & \multicolumn{2}{|c|}{$d(\mathrm{~mm}) \pm 0.5 \mathrm{~mm}$} & \multicolumn{2}{|c|}{$U(\mathrm{~m} / \mathrm{s}) \pm 2.5 \%$} & \multicolumn{2}{|c|}{$\theta\left(^{\circ}\right) \pm 0.2^{\circ}$} \\
\hline & $0 \mathrm{~ms}$ & $20 \mathrm{~ms}$ & $0 \mathrm{~ms}$ & $20 \mathrm{~ms}$ & $0 \mathrm{~ms}$ & $20 \mathrm{~ms}$ \\
\hline 1 & 5 & 1.5 & 22.5 & 20.0 & 0 & -0.4 \\
\hline 2 & 10 & 8.5 & 22.0 & 20.5 & 0 & -1.1 \\
\hline 3 & 15 & 15.0 & 22.0 & 20.5 & 0 & -1.5 \\
\hline 4 & 20 & 23.5 & 21.5 & 20.0 & 0 & 0.2 \\
\hline 5 & 25 & 27.5 & 21.5 & 19.5 & 0 & -0.2 \\
\hline 6 & 30 & 35.0 & 22.5 & 20.0 & 0 & 0.2 \\
\hline 7 & 35 & 42.0 & 21.5 & 19.5 & 0 & 0.4 \\
\hline 8 & 40 & 48.0 & 21.5 & 19.5 & 0 & 1.3 \\
\hline
\end{tabular}


accuracy of the experiments. Although the distance between the projectile and bottom plate changes many times in experiment No0.1, the characteristics are still observed for the cavity around the axisymmetric projectile under the effect of the wall. The analysis indicates that the effects of the interfering factors are within the acceptable range, which allows the observation of the cavity characteristics around the axisymmetric projectile near wall.

\section{References}

Bensow, R.E., Bark, G., 2010. Implicit LES predictions of the cavitating flow on a propeller. ASME J. Fluids Eng. 132, 041302.

Callenaere, M., Franc, J.P., Michel, J.M., et al., 2001. The cavitation instability induced by the development of a re-entrant jet. J. Fluid Mech. 444, 223-256.

Chen, G., Wang, G., Hu, C., et al., 2015. Combined experimental and computational investigation of cavitation evolution and excited pressure fluctuation in a convergent-divergent channel. Int. J. Multiph. Flow 72, 133-140.

De Villiers, E., 2007. The potential of large eddy simulation for the modelling of wall bounded flows. Dep. Mech. Eng. Imp. Coll. Lond., 74-100.

Decaix, J., Goncalvès, E., 2013. Compressible effects modeling in turbulent cavitating flows. Eur. J. Mech. B Fluids 39, 11-31.

Dittakavi, N., Chunekar, A., Frankel, S., 2010. Large eddy simulation of turbulentcavitation interactions in a venturi nozzle. ASME J. Fluids Eng. 132, 121301.

Franc, J.P., Michel, J.M., 2004. Fundamentals of Cavitation. Kluwer Academic, Dordrecht, The Netherlands.

Ganesh, H., 2015. Bubbly Shock Propagation as a Cause of Sheet to Cloud Transition of Partial Cavitation and Stationary Cavitation Bubbles Forming on a Delta Wing Vortex (PH.D.). The University of Michigan, Michigan, USA.

Goncalvès, E., 2011. Numerical study of unsteady turbulent cavitating flows. Eur. Eur. J. Mech. B Fluids 30 (1), 26-40.

Hasuike N., Yamasaki S., Ando J., 2009. Numerical study on cavitation erosion risk of marine propellers operating in wake flow. In: Proceedings of the 7th International Symposium on Cavitation. Nobuhiro Hasuike, Michigan, USA.

He, M., Yuan, L., Zhou, L., et al., 2014. Numerical evaluation of the side wall effect on the flow around a hydrofoil. Eng. Comput. 31 (3), 501-509.

Huang, B., Zhao, Y., Wang, G., 2014. Large eddy simulation of turbulent vortexcavitation interactions in transient sheet/cloud cavitating flows. Comput. Fluids 92, $113-124$.

Ji, B., Luo, X., Wang, X., et al., 2011. Unsteady numerical simulation of cavitating turbulent flow around a highly skewed model marine propeller. ASME J. Fluids Eng. 133, 011102.

Ji, B., Luo, X., Peng, X., et al., 2012. Numerical analysis of cavitation evolution and excited pressure fluctuation around a propeller in non-uniform wake. Int. J. Multiph. Flow 43, 13-21.

Ji, B., Luo, X., Peng, X., et al., 2013. Three-dimensional large eddy simulation and vorticity analysis of unsteady cavitating flow around a twisted hydrofoil. J. Hydrodyn. 25 (4), 510-519.

Ji, B., Luo, X.W., Arndt, R.E.A., et al., 2015. Large Eddy simulation and theoretical investigations of the transient cavitating vortical flow structure around a NACA66 hydrofoil. Int. J. Multiph. Flow 68, 121-134.

Kawanami, Y., Kato, H., Yamaguchi, H., et al., 1997. Mechanism and control of cloud cavitation. ASME J. Fluids Eng. 119, 788-794.

Kubota, A., Kato, H., Yamaguchi, H., et al., 1989. Unsteady structure measurement of cloud cavitation on a foil section using conditional sampling technique. ASME J. Fluids Eng. 111, 204-210.

Kunz, R.F., Boger, D.A., Stinebring, D.R., et al., 2000. A preconditioned Navier-Stokes method for two-phase flows with application to cavitation prediction. Comput. Fluids $29(8), 849-875$.

Lu, N.X., Bensow, R.E., Bark, G., 2010. LES of unsteady cavitation on the delft twisted foil. J. Hydrodyn. Ser. B 22 (5), 784-791.

Lu, N.X., Bensow, R.E., Bark, G., 2014. Large Eddy simulation for incompressible flows simulation of cavitation development on highly skewed propellers. J. Mar. Sci. Technol. 19 (2), 197-214.

Ma, J., Hsiao, C.T., Chahine, G.L., 2017. A physics based multiscale modeling of cavitating flows. Comput. Fluids 145, 68-84.

Ma, J., Oberai, A.A., Drew, D.A., et al., 2010. A quantitative sub-grid air entrainment model for bubbly flows-plunging jets. Comput. Fluids 39 (1), 77-86.
Nicoud, F., Ducros, F., 1999. Subgrid-scale stress modelling based on the square of the velocity gradient tensor. Flow Turbul. Combust. 62 (3), 183-200.

Passandideh-Fard, M., Roohi, E., 2008. Transient simulations of cavitating flows using a modified volume-of-fluid (VOF) technique. Int. J. Comput. Fluid Dyn. 22 (1-2), 97-114.

Roohi, E., Zahiri, A.P., Passandideh-Fard, M., 2013. Numerical simulation of cavitation around a two-dimensional hydrofoil using VOF method and LES turbulence model. Appl. Math. Model. 37 (9), 6469-6488.

Sahner J., Weinkauf T., Hege H.C., 2005. Galilean invariant extraction and iconic representation of vortex core lines. In: Proceedings EUROGRAPHICS-IEEE VGTC Symposium on Visualization(2005), Leeds, United Kindom.

Schmitt, F.G., 2007. About boussinesq's turbulent viscosity hypothesis: historical remarks and a direct evaluation of its validity. Comptes Rendus Mécanique 335 (9), 617-627.

Seo, J.H., Moon, Y.J., Shin, B.R., 2008. Prediction of cavitating flow noise by direct numerical simulations. J. Comput. Phys. 227 (13), 6511-6531.

Soyama H., Kato H., Oba R., 1992. Cavitation observation of severely erosive vortex cavitation arising in a centrifugal pump. In: Proceedings of the Third IMechE International Conference on Cavitation, pp. 103-110.

Temmerman, L., Leschziner, M.A., Mellen, C.P., et al., 2003. Investigation of wallfunction approximations and subgrid-scale models in large eddy simulation of separated flow in a channel with streamwise periodic constrictions. Int. J. Heat Fluid Flow 24 (2), 157-180.

Wang, G., Ostoja-Starzewski, M., 2007. Large eddy simulation of a sheet/cloud cavitation on a NACA0015 hydrofoil. Appl. Math. Model. 31 (3), 417-447.

Wang, Y., Liao, L., Du, T., et al., 2014. A study on the collapse of cavitation bubbles surrounding the underwater-launched projectile and its fluid-structure coupling effects. Ocean Eng. 84, 228-236.

Wang Y., Wu X., Huang C., et al., 2015. On the flow structure of cloud cavitating flow around an axisymmetric body near the free surface. Journal of Physics: Conference Series. IOP Publishing 656(1), p. 012174.

Wang, Y., Huang, C., Fang, X., et al., 2015b. On the cloud cavitating flow over a submerged axisymmetric projectile and comparison between 2D RANS and 3d LES methods. ASME J. Fluids Eng. 138, 061102.

Wang, Y., Huang, C., Fang, X., et al., 2016a. Cloud cavitating flow over a submerged axisymmetric projectile and comparison between two-dimensional RANS and threedimensional large-Eddy simulation methods. ASME J. Fluids Eng. 138, 061102.

Wang, Y., Wu, X., Huang, C., et al., 2016b. Unsteady characteristics of cloud cavitating flow near the free surface around an axisymmetric projectile. Int. J. Multiph. Flow $85,48-56$.

Watanabe T., Kawamura T., Takekoshi Y., , et al.2003. Simulation of steady and unsteady cavitation on a marine propeller using a RANS CFD code. In: Proceedings of the Fifth International Symposium on Cavitation. Osaka, Japan.

Wu, X.C., Wang, Y.W., Huang, C.G., et al., 2016. Effect of mesh resolution on large eddy simulation of cloud cavitating flow around a three dimensional twisted hydrofoil. Eur. J. Mech. B-Fluids 55, 229-240.

Xin, C., LU, C., Jie, L.I., et al., 2008. The wall effect on ventilated cavitating flows in closed cavitation tunnels. J. Hydrodyn. Ser. B 20 (5), 561-566.

Ying, C., Lu, C.J., 2008. A homogenous-equilibrium-model based numerical code for cavitation flows and evaluation by computation cases. J. Hydrodyn. Ser. B 20 (2), 186-194.

Yu, C., Wang, Y., Huang, C., et al., 2017. Large eddy simulation of unsteady cavitating flow around a highly skewed propeller in nonuniform wake. ASME. J. Fluids Eng. 139, 041302-041310.

Yu, X.X., Huang, C.G., Du, T.Z., et al., 2014. Study of characteristics of cloud cavity around axisymmetric projectile by large Eddy simulation. ASME J. Fluids Eng. 136, 051303.

Zhou, L., Wang, Z., 2008. Numerical simulation of cavitation around a hydrofoil and evaluation of a RNG $\kappa-\varepsilon$ model. ASME J. Fluids Eng. 130, 011302.

Zhou, J.J., Yu, K.P., Min, J.X., et al., 2010. The comparative study of ventilated super cavity shape in water tunnel and infinite flow field. J. Hydrodyn. Ser. B 22 (5), 689-696. 